\title{
Article \\ A Quantum Weak Signal Detection Method for Strengthening Target Signal Features under Strong White Gaussian Noise
}

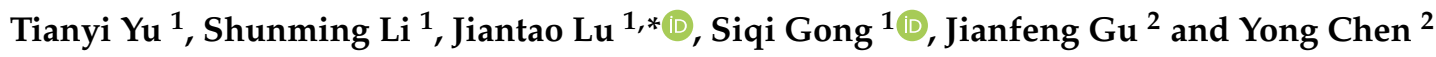 \\ 1 College of Energy and Power Engineering, Nanjing University of Aeronautics and Astronautics, \\ Nanjing 210016, China; tianyi_yu@nuaa.edu.cn (T.Y.); smli@nuaa.edu.cn (S.L.); gongsiqi07@nuaa.edu.cn (S.G.) \\ 2 Jiangsu Donghua Testing Technology Co., Jingjiang 214519, China; gjf@dhtest.com (J.G.); \\ chenyong@dhtest.com (Y.C.) \\ * Correspondence: lujt@nuaa.edu.cn; Tel.: +86-15191452453
}

check for updates

Citation: Yu, T.; Li, S.; Lu, J.; Gong, S.; $\mathrm{Gu}$, J.; Chen, Y. A Quantum Weak Signal Detection Method for Strengthening Target Signal Features under Strong White Gaussian Noise. Appl. Sci. 2022, 12, 1878. https:// doi.org/10.3390/app12041878

Academic Editor: Amerigo Capria

Received: 20 December 2021

Accepted: 31 January 2022

Published: 11 February 2022

Publisher's Note: MDPI stays neutral with regard to jurisdictional claims in published maps and institutional affiliations.

Copyright: () 2022 by the authors Licensee MDPI, Basel, Switzerland. This article is an open access article distributed under the terms and conditions of the Creative Commons Attribution (CC BY) license (https:// creativecommons.org/licenses/by/ $4.0 /)$.

\begin{abstract}
As the noise power increases, the target signal features become less obvious, which leads to the failure of weak signal detection methods. To address this problem, a quantum weak signal detection method, Local Semi-Classical Signal Analysis-Singular Value Decomposition (LSCSA-SVD), for strengthening target signal features under strong white Gaussian noise is proposed. Firstly, the time domain weak signal is quantized by the Schrodinger operator and its discrete spectrum formula. Then, in the quantum domain, the later eigenvalues are used to reconstruct the time domain signal, which can protect and enhance the target signal features. Finally, the difference between signal and noise in the singular value vector is used to further extract the reconstruction signal features. In simulation, the LSCSA-SVD can accurately extract target signals from white Gaussian noise signals with a signal-to-noise ratio (SNR) of $-30 \mathrm{~dB}$, which is better than the comparison methods. In the experiment, the weak acceleration sensor signal and the weak signal of the test circuit are successfully extracted. The results show that the LSCSA-SVD can suppress strong noise and improve the SNR.
\end{abstract}

Keywords: weak signal detection; quantum signal processing; signal features; local semi-classical signal analysis; SVD; bearing fault features

\section{Introduction}

A weak signal is a signal in which the amplitude of the target signal is smaller than that of the noise. The signal-to-noise ratio (SNR) of a weak signal is less than 0 . The target signal is completely hidden in the strong noise, whose features are fuzzy and difficult to extract [1-3]. Therefore, the key to weak signal detection (WSD) is to protect the target signal while eliminating the noise. The formula for calculating SNR in this paper is as follows [4]:

$$
\text { SNR }=10 \log \frac{\text { Signal power }}{\text { Noise Power }}
$$

With the development of integrated circuits, the precision instrument industry has a higher demand for weak signal detection [5,6]. A complex marine environment forms natural strong noise, which limits the transmission distance of sonar information $[7,8]$. Weak faults of rotating machinery (e.g., strong noise, early fault) are difficult to diagnose $[9,10]$. The above are the WSD problems that need to be solved urgently. Effectively solving WSD problems can promote the development of high and new technology.

Researchers have carried out a variety of weak signal detection studies according to the needs of industries from the perspectives of the type and intensity of noise, the type of target signal, and the signal processing methods. The amplitude distribution of White Gaussian Noise (WGN) obeys Gaussian distribution, and its power spectral density obeys uniform distribution [11,12]. With components all over the spectrum, WGN is a kind of simulation noise suitable for signal-noise separation. In recent years, the influence of WGN has been considered in many fields-for example, the influence of time delay and noise 
on the electrical activity of neurons [13], noise suppression ability of the scale-free spiking neural network (SFSNN) with high and low average clustering coefficients (ACC) under WGN [14], the application of Gaussian approximation in gene expression models [15], the combined effects of phase and WGN in the performance of radio over fiber-orthogonal frequency division multiplexing (ROF-OFDM) schemes [16], WGN image denoising based on edge detection [17], and so on. The abovementioned research shows that WGN as simulation signal applies to physical, chemical and biological systems well. Owing to the wide applicability of WGN, the weak signal detection algorithm of WGN will be studied in this paper.

When the noise intensity is higher than the target signal, some traditional signal processing methods fail. Researchers have proposed many methods for weak signal detection, such as the following: multiple autocorrelation algorithm [18,19], Singular Value Decomposition (SVD) [20,21], convolutional neural network (CNN) [22-24], etc. A multiple autocorrelation algorithm relies on the principle of strong autocorrelation of the target signal and weak autocorrelation of random noise, and extracts the target signal through multiple autocorrelation calculation of the weak signal [18]. After using multiple autocorrelation, the signal amplitude attenuation is serious and difficult to recover. With the increase of noise power, the autocorrelation of the target signal is gradually weakened. This leads to the failure of multiple autocorrelation to extract signals with an SNR of less than $-20 \mathrm{~dB}$ [19]. SVD is used to decompose the weak signal vector space into two independent subspaces composed of the target signal and strong noise. The denoising of SVD is realized by eliminating the strong noise subspace [20]. When the noise power is increased, the boundary between the target signal features and the noise gradually becomes blurred. The two subspaces are difficult to separate from each other, which leads to the weakening of the denoising ability of SVD [21]. According to the literature mentioned above, the strength of target signal features directly affects the denoising ability of the algorithm. Therefore, the protection and enhancement of target signal features will be fully considered in this paper.

Quantum signal processing (QSP) is an idea that applies the mathematical framework of quantum mechanics to signal processing $[25,26]$. There have been a number of studies on QSP in recent years. Le Yang proposed a compound quantum-inspired structural element (CQSEE) for erosion used to extract fault information from vibration signals of mechanical transmission systems [27]. Chao-Yang Pang et al., proposed quantum discrete Fourier transform [28]. Yan-Long Chen et al., proposed a denoising method for mechanical vibration signals based on quantum superposition state parameter estimation and dual tree complex wavelet [29]. Taous-Meriem et al., proposed a semi-classical signal analysis (SCSA) for blood pressure detection and magnetic resonance spectroscopy detection [30]. Raphael Smith et al., proposed an image noise reduction method based on quantum adaptive dictionary learning [31]. The abovementioned literature has certain effects in the denoising of signals and images in the mechanical and medical fields. But the research of quantum signal processing in weak signal detection is still a blank state.

In the signal, the relation between the target signal and the noise is similar to the two states in the superposition principle of quantum states. The operators are used to interfere with quantum states in superposition states, and the probability amplitude of the two quantum states are changed, which make one quantum state easier to be observed. By the above principle, the strong and weak relationship between the target signal and the noise can be changed. Weak signal denoising realized by this method can avoid the influence of strong noise to the greatest extent. Not only that, but quantum states have parallel computing capabilities. The simultaneous operation of $\mathrm{n}$ quantum states (signal points) can accelerate the overall running speed and shorten the running time. In addition, there are many unknown properties in the quantum domain to be studied. To sum up, QSP has many possibilities in the field of weak signal detection.

Aiming at these abovementioned problems, a quantum weak signal detection method, Local Semi-Classical Signal Analysis- Singular Value Decomposition (LSCSA-SVD), is proposed for the protection and enhancement of target signal features and the possibility 
of QSP in WSD. First, LSCSA is used to enhance the features, and then SVD is used to further extract weak target signals. In the simulation and experiment, the effectiveness of LSCSA-SVD is verified by comparison methods. The main contributions of this paper are as follows:

1. A quantum signal processing method, Local Semi-Classical Signal Analysis (LSCSA), has been proposed. In the LSCSA, Schrodinger operator and discrete spectrum formula are used to transform a time domain weak signal into a quantum domain. Then the quantum domain signal can be restored to the time domain signal by reconstructing the formula. The quantization ability of LSCSA is the basis of studying the characteristics of weak signals in the quantum domain.

2. The quantum domain characteristics and the singular value characteristics of weak signals have been studied. In the quantum domain, the weak target signal mainly exists in the later portion of the eigenvalue sequence (detail region). The weak target signal can be located in strong noise only by using a detailed region reconstruction signal. The weak target signal features can be protected and strengthened by this characteristic. In the singular value vector, the singular values representing the weak target signal and the strong noise signal components can be separated as separate coordinates. By this characteristic, noise and target signals can be accurately separated.

3. A quantum weak signal detection algorithm, the LSCSA-SVD, has been proposed in this paper. The weak signal features are protected and strengthened by LSCSA, which solve the problem that SVD cannot detect weak signals when the signal features are not obvious. SVD is used to further extract the signal features of LSCSA reconstruction signal, which solve the problem that LSCSA cannot extract signal features directly. LSCSA-SVD can detect weak signals with low SNR and improve the SNR.

4. A clear guideline on how to select the optimal parameters of the LSCSA-SVD has been given.

The rest of this paper is organized as follows: In Section 2, the theory of the QSP, the LSCSA, and the singular value characteristics of weak signals will be introduced. In Section 3, the LSCSA-SVD and how to select the optimal parameters of the LSCSA-SVD will be introduced. In Section 4, the LSCSA-SVD will be verified on the simulation signal (SNR $=-30 \mathrm{~dB})$. In Section 5 , the LSCSA-SVD will be analyzed experimentally with experimental test data. Section 6 will summarize the full text.

\section{Materials and Methods}

\subsection{Quantum Signal Processing and LSCSA}

Quantum signal processing (QSP) is an idea that applies the mathematical framework of quantum mechanics to signal processing [32]. QSP is a natural simulation algorithm framework that builds a new algorithm based on quantum mechanics and improves the existing algorithm. The traditional quantum computation uses the quantum effect of the physical entity to complete the corresponding processing, but this method is limited by the axioms and constraints of quantum physics, and it is usually difficult to realize. QSP only uses the mathematical framework and ideas of quantum mechanics and establishes corresponding processing algorithms based on the axioms in quantum mechanics. This method is not materially limited by the laws of quantum physics, and its implementation entity is an ordinary computer system [33].

In quantum mechanics, a (non-relativistic) particle in a potential state is described by a wave function $\psi$, whose squared absolute value $|\psi|^{2}$ corresponds to the probability of presence of the particle [34]. The normalization of probability implies that $\int|\psi|^{2}=1$, and the wave function belongs to the Hilbert space of functions with bounded integrals ( $\mathrm{L}^{2}$ norm).

The difficulty of QSP lies in how to combine the signal processing problem with the mathematical framework of quantum mechanics. In QSP, the relationship between the signal point and the time period are similar to the relationship between a particle and the potential space, and the state of the signal point in the time period (the amplitude of the signal) is similar to the state of a particle in the potential space (the potential of the 
particle). Referring to the concept of wave function, Local Semi-Classical Signal Analysis (LSCSA) replaces the particle in the potential space as each point of a 1-dimensional signal, and the amplitude of each signal point is used to replace the potential of the particle. The Schrödinger operator $\Phi$ and its discrete spectral formula are the bridge between the time domain and the quantum domain. The calculation process is called the quantization process of the time-domain signal. The result/space of the computation is called the quantum domain.

In LSCSA, the Schrödinger operator $\Phi$ and its discrete spectral formula are used to decompose and reconstruct the 1-dimensional signals $y$. The discrete spectrum formula is as follows [35-37]:

$$
H_{h}(y) \Phi=-\frac{\hbar^{2}}{2 m} \nabla^{2} \Phi-V(r) \Phi, \Phi \in H^{2}(\mathbb{R})
$$

where $H_{h}(y)$ is the discrete spectrum of Schrödinger operators, $\hbar$ is the Planck constant, $m$ is the particles in the potential of quality, $\nabla^{2} \Phi$ is Laplacian operator, $V(r)$ is the potential Schrödinger operators, and $H^{2}(\mathbb{R})$ denotes the Sobolev space of order 2 . In order to simplify the formula, $\frac{\hbar^{2}}{2 m}$ is replaced with constant $h^{2}, \nabla^{2} \Phi$ is replaced by the second derivative of Schrödinger operators, and the potential of Schrödinger operators $V(r)$ is replaced by 1-dimensional vibration signal $y$. The simplified formula can be written as

$$
H_{h}(y) \Phi=-h^{2} \frac{d^{2} \Phi}{d t^{2}}-y \Phi, \Phi \in H^{2}(\mathbb{R}), h>0
$$

According to the Fourier spectrum method [38], the second derivative of Schrödinger operators can be written as Schrödinger operators $\Phi$ multiplied by the spectrum derivative matrix $D$. Equation (3) can be rewritten into the following form:

$$
H_{h}(y) \Phi=\left[-h^{2} \boldsymbol{D}-\operatorname{diag}(y)\right] \Phi
$$

The discrete spectrum $H_{h}(y)$ of Schrödinger operator is calculated by Equation (4). The eigenvalues and eigenvectors of the spectrum are calculated by the following formula:

$$
H_{h}(y) \psi(t)=\lambda \psi(t)
$$

where the eigenvalues of $H_{h}(y)$ are $\lambda$ and the eigenfunctions are $\psi(t)$. The negative eigenvalues of $H_{h}(y)$ are denoted as $-k_{n h}{ }^{2}$. The number of the negative eigenvalues is denoted as $N_{h}$. The associated $\mathrm{L}^{2}$-normalized eigenfunctions are denoted as $\psi_{n h}, n=1, \ldots, N_{h}$. The negative eigenvalues and the corresponding square eigenfunctions are used for reconstruction. The reconstructed signal is denoted as $y h$, and the reconstruction formula is as follows:

$$
y_{h}(t)=4 h \sum_{n}^{N_{h}} k_{n h} \psi^{2} \mathrm{nh}(t), t \in \mathbb{R}
$$

The flow chart of the LSCSA is shown in Figure 1. The steps of LSCSA are as follows.

1. The Schrodinger operator $\Phi$ is used to calculate the discrete spectrum $H_{h}(y)$ of the time-domain weak signal $y(t)$. The time-domain weak signal $y(t)$ is converted to a quantum domain signal.

2. Calculate eigenvalues $-k_{n h}{ }^{2}$ and eigenvectors $\psi(t)$ of the discrete spectrum $H_{h}(y)$.

3. In the quantum domain, the target signal is hidden in the eigenvalues sequence. Select the appropriate eigenvalues from the sequence $-k_{n h}^{2}$.

4. The appropriate negative eigenvalues and eigenvectors are used for signal reconstruction. The quantum domain signal is restored to the time domain signal $y_{h}(t)$. 


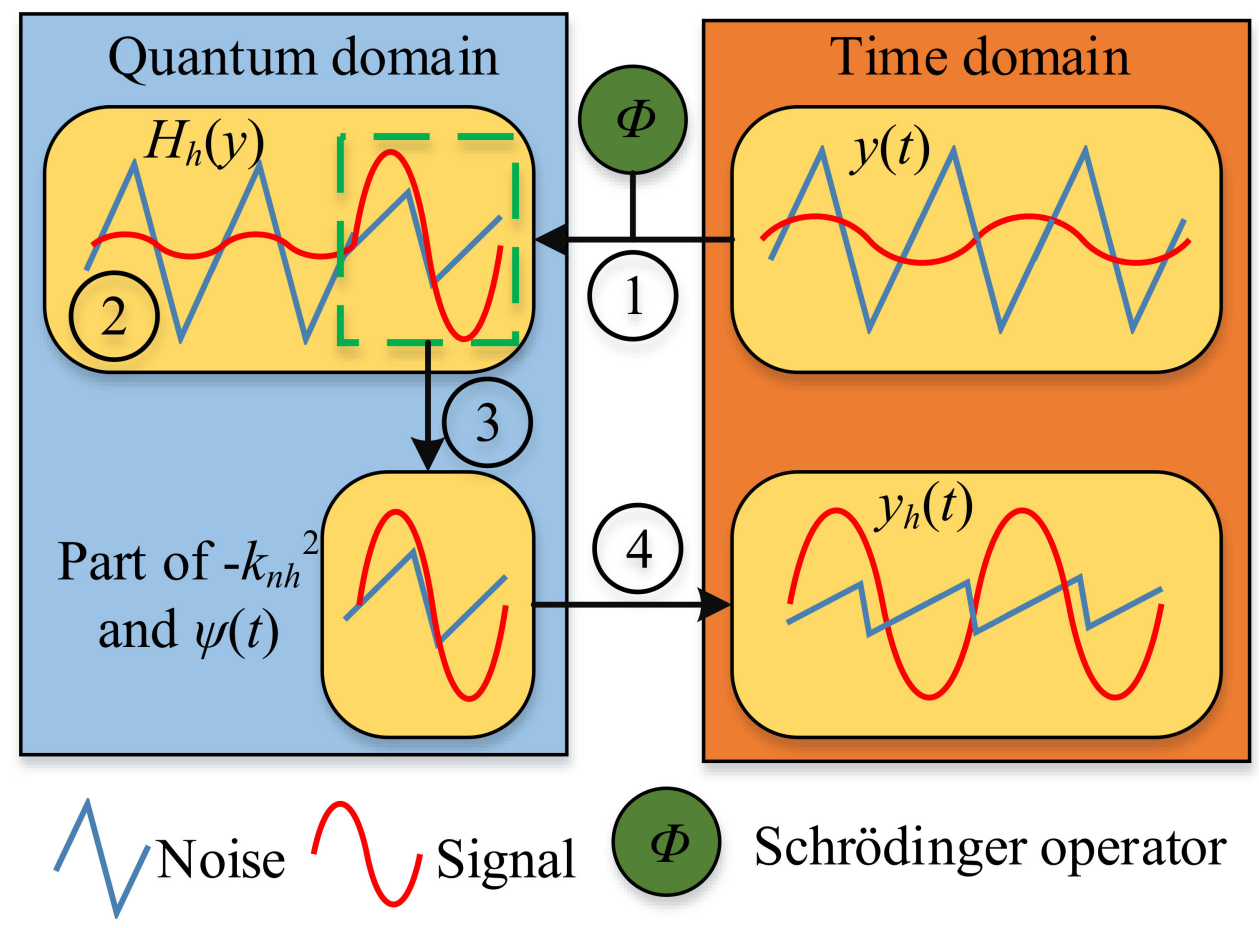

Figure 1. Flow chart of the LSCSA.

\subsection{Quantum Domain Characteristics of Weak Signal}

In signal processing, it is a common method to transform the time-domain signal to other domains for analysis. The same goes for the LSCSA. In Figure 1, the time-domain weak signal $y(t)$ is converted to a quantum-domain signal. In the quantum domain, some characteristics of the weak signal are different with the time domain.

In the LSCSA, parameter $h$ plays an important role. When $h$ decreases, $N_{h}$ increases and the reconstructed signal $y_{h}$ is closer to the original signal $y$. When the parameter $h$ satisfies Equation (7) and $n=1, y_{h}=y$,

$$
4 h \sum_{n=1}^{N_{h}} k_{n h}=\int_{-\infty}^{+\infty} y(t) d t
$$

when $y \in \mathrm{L}^{1 / 2}(\mathbb{R})$,

$$
\lim _{h \rightarrow 0} h N_{h}=\frac{1}{\pi} \int_{-\infty}^{+\infty} \sqrt{y(t)} d t
$$

It can be seen that $N_{h}$ is a decreasing function of $h$. With the decrease of $h$, the number of negative eigenvalues and corresponding eigenfunctions increases. The negative eigenvalue $-k_{n h}{ }^{2}$ is an ascending sequence from small to large. The front of the negative eigenvalues sequence (e.g., $\psi_{1 h}, \psi_{2 h}$ ) depicts main profile characteristics of signal y [30]. With the increase of $N_{h}$, the eigenvalue depicts the signal $y$ details characteristics. Eigenvectors contain more and more information.

Based on the characteristics of the LSCSA in the quantum domain, the following inference follows. For the ordinary signal, the noise is smaller than the target signal. In the ordinary signal, the noise belongs to the detail part. By adjusting the $h$ value, the signal can be reconstructed with a small number of negative eigenvalues to achieve the purpose of noise reduction. However, for weak signals, the roles of noise and signal are reversed. The target signal annihilated by strong noise is hidden in the details of the weak signal.

By using these characteristics, the target signal can be located by adjusting the $h$ value and using the back of the negative eigenvalue sequence. The confusion between target signal and strong noise can be eliminated. The interference ability of the strong noise is greatly reduced. The target signal will not be considered as noise during signal processing, 
and can be protected by the LSCSA. After using the LSCSA, the target signal features can be strengthened.

\subsection{Singular Value Characteristics of Weak Signal}

As a traditional signal processing method, Singular Value Decomposition (SVD) has limitations [20]. When the target features in the signal are relatively obvious, SVD has a better extraction effect on the signal features. On the contrary, SVD has little effect on signal improvement. It means that weak signals with signal features submerged in strong noise are not suitable for SVD to extract target signals.

It is mentioned in Section 2.1 that after using the LSCSA, the target signal can be located in the weak signal and the target signal features can be strengthened, which ensures that the target characteristics have been obviously extracted from the reconstructed signal of the LSCSA. Thus, the signal can be improved by SVD after the LSCSA.

The one-dimensional vector should be constructed as a matrix before using SVD. Let the one-dimensional discrete signal $Y=\left\{y_{1}, \ldots, y_{k}\right\}$, and the matrix construction form is as follows:

$$
\boldsymbol{H}=\left(\begin{array}{cccc}
y_{1} & y_{2} & \cdots & y_{j} \\
y_{2} & y_{3} & \cdots & y_{j+1} \\
\vdots & \vdots & \vdots & \vdots \\
y_{k-j+1} & y_{k-j+2} & \cdots & y_{k}
\end{array}\right)
$$

where $1<j<k$, let $m=k-j+1, H \in \mathbf{R}^{m \times j}$. This matrix is the Hankel matrix. Signal $Y$ contains two components: target signal $S$ and noise signal $N$. Target signal $S$ contains linear signal $S_{l}$ and nonlinear periodic signal $S_{p}$ (the above linear signal and nonlinear signal refer to the time-domain image shape, the same as the linear function and nonlinear function signal in a mathematical concept). Signal $Y$ can be expressed in the following form:

$$
Y=S+N=S_{l}+S_{p}+N
$$

After signal $Y$ is constructed as Hankel matrix $\boldsymbol{H}$, it can be expressed in the following form:

$$
\boldsymbol{H}=\boldsymbol{H}_{S}+\boldsymbol{H}_{N}=\boldsymbol{H}_{l}+\boldsymbol{H}_{p}+\boldsymbol{H}_{N}
$$

where $\boldsymbol{H}_{S}, \boldsymbol{H}_{N}, \boldsymbol{H}_{l}, \boldsymbol{H}_{p}$ are Hankel matrices constructed by $S, N, S_{l}, S_{p}$, respectively.

Characteristics of Hankel matrices: The next line of vectors lags only one data point behind the previous line.

Characteristics of SVD: The number of non-zero singular values generated after SVD of the matrix is equal to the rank of the matrix.

The following conclusions can be drawn from the above two characteristics:

1. In the matrix $\boldsymbol{H}_{l}$ constructed by linear signal $S_{l}$, each row is linearly dependent. So the rank of the matrix $\boldsymbol{H}_{l} r=1$, and the singular value vector can be expressed as $\sigma\left(\boldsymbol{H}_{l}\right)=$ $\left(\sigma_{l}, 0, \ldots, 0\right)$.

2. Due to the periodicity of the nonlinear periodic signal $S_{p}$, denoted by period $T$, the vector of the first $T$ row of the matrix $\boldsymbol{H}_{p}$ is linearly independent, and the other lines are linearly related to the vector of the first $T$ row. Therefore, the rank of the matrix $\boldsymbol{H}_{p} r<\min (m, j)$, and the singular value vector can be expressed as $\sigma\left(\boldsymbol{H}_{p}\right)=\left(\sigma_{p 1}, \sigma_{p 2}\right.$, $\left.\ldots, \sigma_{p r}, 0, \ldots, 0\right)$.

3. The random noise signal $N$ is irrelevant at every moment. Therefore, each row of the constructed matrix $\boldsymbol{H}_{N}$ is unrelated, so the rank of the matrix $\boldsymbol{H}_{N} r=\min (m, j)$. The singular value vector can be expressed as $\sigma\left(\boldsymbol{H}_{N}\right)=\left(\sigma_{n 1}, \sigma_{n 2}, \ldots, \sigma_{n r}\right)$.

Reference [39] points out that when $\min (m, j)$ is maximized, the singular value of matrix $\boldsymbol{H}$ has a relationship with the singular value of matrix $\boldsymbol{H}_{N}, \boldsymbol{H}_{l}, \boldsymbol{H}_{p}$, as follows [39]:

$$
\sigma(\boldsymbol{H})=\sigma\left(\boldsymbol{H}_{l}+\boldsymbol{H}_{p}+\boldsymbol{H}_{N}\right) \approx\left(\sigma_{l}, \sigma_{p 1}, \sigma_{p 2}, \ldots, \sigma_{p r}, \sigma_{n 1}, \ldots, \sigma_{n r}\right)
$$


It can be seen that, in the total singular value vector of mixed signals, the singular values representing the target signal and the noise signal components can be separated as separate coordinates. Moreover, the singular value of the noise component will be separated into the posterior coordinates of the total vector. Therefore, the signal noise reduction can be achieved by selecting the appropriate singular value and its corresponding vector for SVD reconstruction.

\section{LSCSA-SVD}

According to the theory in Section 2, a novel quantum weak signal detection method, LSCSA-SVD, is proposed. The steps of LSCSA-SVD (Algorithm 1) are as follows, and the flow diagram is shown in Figure 2.
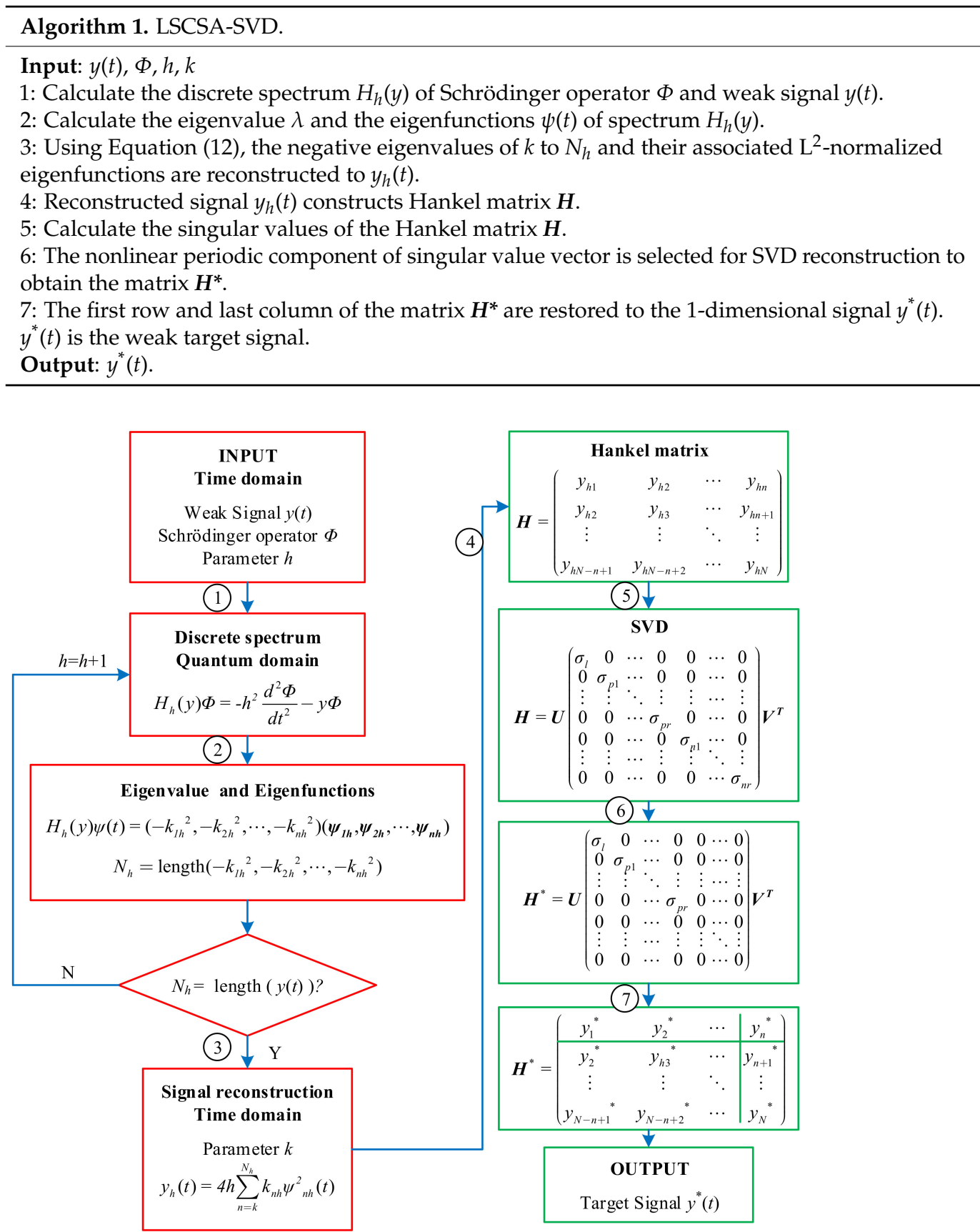

Figure 2. Flow diagram of LSCSA-SVD. 


$$
y_{h}(t)=4 h \sum_{n=k}^{N_{h}} k_{n h} \psi^{2}{ }_{n h}(t), t \in \mathbb{R}
$$

There are two important parameters, $h$ and $k$, in the LSCSA-SVD. They can be selected in the following manner:

\section{Parameter $h$}

Standard 1: $N_{h}=$ length $(y(t))$.

Standard 2: On the premise of satisfying Standard 1, the parameter $h$ should be as large as possible.

From Equation (4), it can be seen that the dimension of discrete spectrum $H_{h}(y)$ is equal to the length of signal $y$, and $N_{h} \leq$ length $(y(t))$. In order to obtain as many detailed features in the reconstructed signal as possible, as many negative eigenvalues as possible are needed. So $N_{h}=$ length $(y(t))$ (Standard 1). While satisfying Standard 1, if the parameter $h$ is too small, the reconstructed signal will be seriously distorted. Therefore, the parameter $h$ needs to be as large as possible (Standard 2). Parameter $h$ can be obtained through the loop on the left of Figure 2.

\section{2. $\quad$ Parameter $k$}

Parameter $k$ will be selected in combination with simulation signals.

Select target signal $x(t)=2 \sin \left(2^{*} \pi^{*} 50^{*} t\right)$. The sampling point is 1500 . The sampling frequency is $1000 \mathrm{~Hz}$. The simulation signal $y(t)$ is $x(t)$ superimposed with Gaussian white noise $(\mathrm{SNR}=-30 \mathrm{~dB})$. The time domain and frequency domain diagram of $y(t)$ are shown in Figure 3.

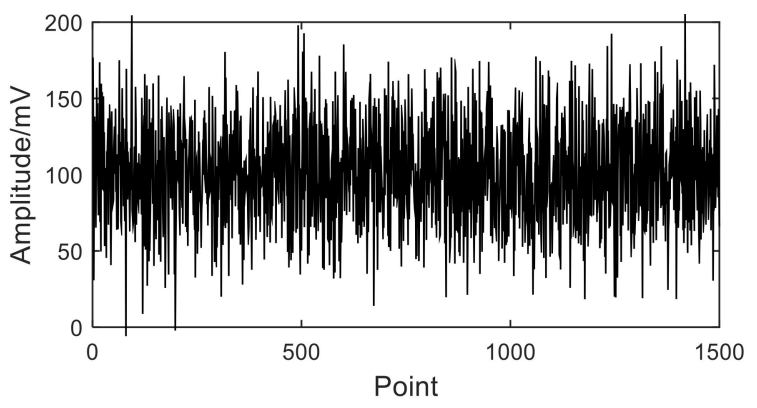

(a)

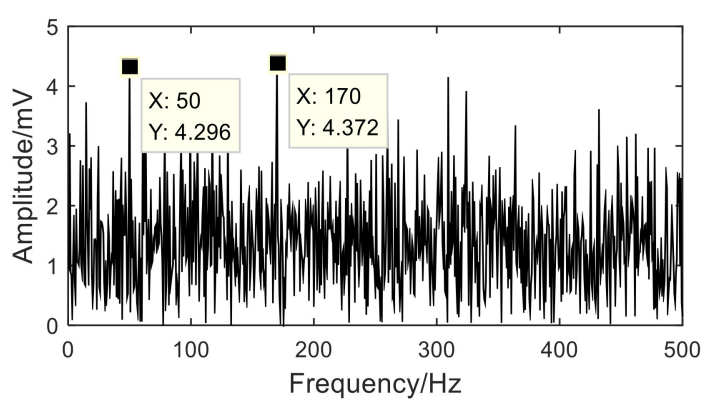

(b)

Figure 3. Simulated signal $y(t)$ (a) Time domain (b) Frequency domain.

In Figure 3a, the amplitude of the simulation signal is higher than $100 \mathrm{mV}$, while the target signal is a sinusoidal signal with amplitude of $2 \mathrm{mV}$. In Figure $3 \mathrm{~b}$, the peak frequency is $170 \mathrm{~Hz}$. The second peak, $50 \mathrm{~Hz}$, is the target signal frequency, and other frequencies are very prominent. Therefore, the target signal has been completely annihilated by strong noise.

Calculate the spectrum $H_{h}(y)$ of $y(t)$, and the parameter $h=0.89$. Calculate the eigenvalues and eigenfunctions of $H_{h}(y)$. In this paper, the parameter $k$ is selected according to the negative eigenvalue $k_{n h}$. The curve of $k_{n h}$ is shown in Figure 4.

According to the LSCSA theory in Section 2.2, the area with sharp change in the blue box in Figure 4 is the main contour feature area of the signal, the area with sharp change in the red box in Figure 4 is the detail feature area of the signal, and the area with moderate change in Figure 4 without a box is the transition area. The red region is the target region selected by the LSCSA. The parameter $k$ should be the intersection point of the transition zone and the detail zone. $k_{n h}$ is a series of discrete points. In order to select parameter $k$ better, $k_{n h}$ is smoothly fitted. The fitted curve is denoted as $k_{n h}+$, as shown in Figure 5 . The differential of $k_{n h}+$ is shown in Figure 6. 


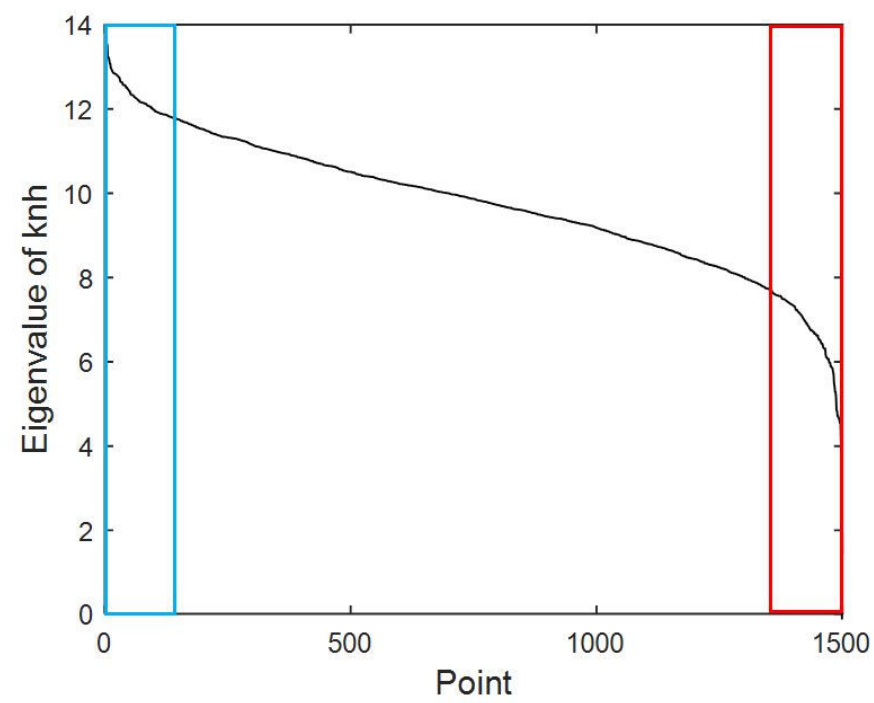

Figure 4. The curve of $k_{n h}$.

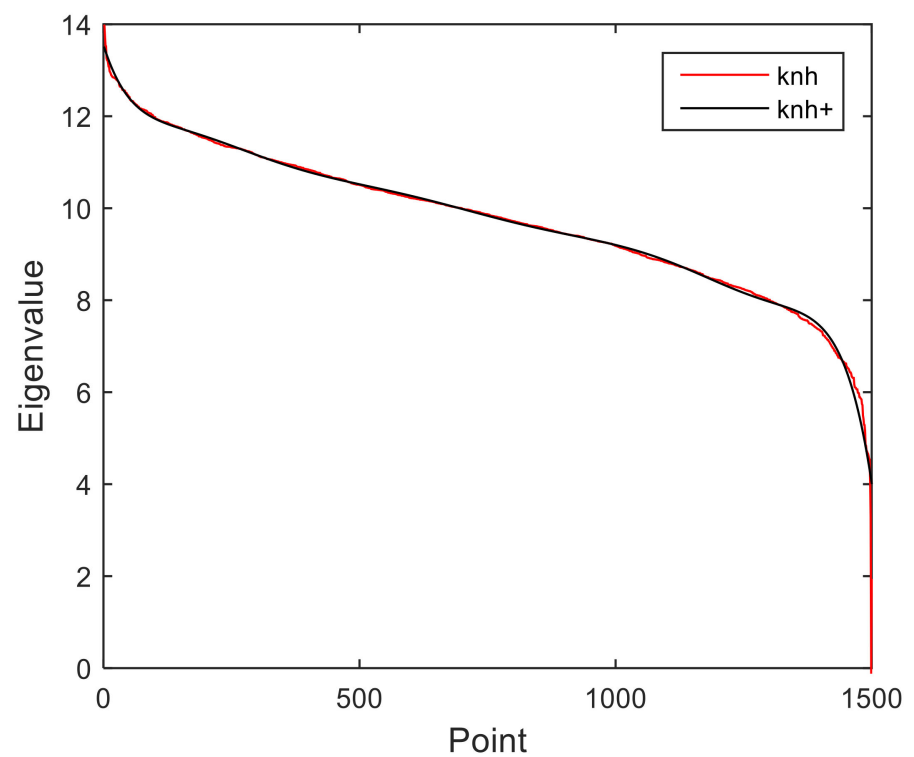

Figure 5. The curves of $k_{n h}$ and $k_{n h}+$.

In Figure 6, the intersection of the transition zone and the detailed feature zone can be clearly found. The coordinate of the peak point of the rightmost crest is the value of parameter $k$. Because of the smooth fitting process, in order to avoid errors, the coordinates in the $k \pm 10$ neighborhood can be used as the value of parameter $k$. The selection rule of parameter $k$ can be summarized as the following steps:

1. Calculate the discrete spectrum $H_{h}(y)$ and parameter $h$ of the signal $y(t)$.

2. Calculate the eigenvalues $-k_{n h}{ }^{2}$ of the discrete spectrum $H_{h}(y)$.

3. Fit the discrete point $k_{n h}$ into a curve $k_{n h}+$.

4. Calculate the differentiation of $k_{n h}+$ and draw the graph.

5. The abscissa value of the rightmost peak point in the differential curve of $k_{n h}+$ is the value of parameter $k$. 


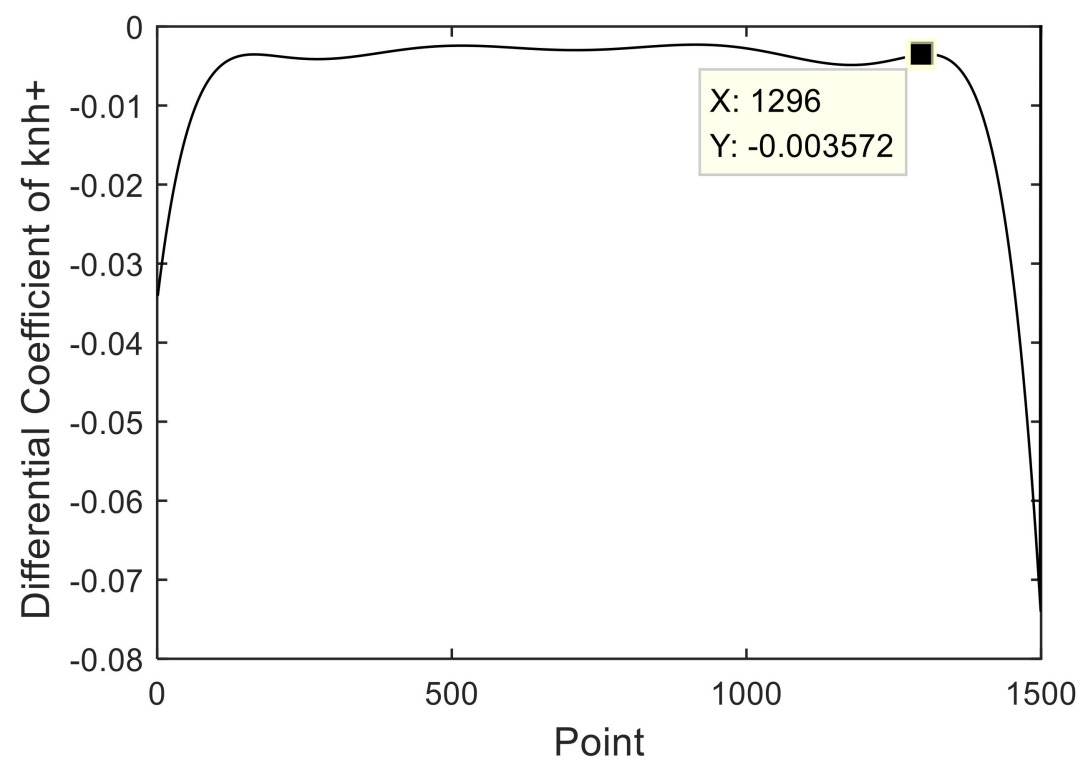

Figure 6. The differential curve of $k_{n h}+$.

In this paper, the parameter $k$ of the simulation signal is 1296.

\section{Simulation}

The results of LSCSA for the simulation signal in Section 3 are shown in Figure 7. Owing to a lot of noise around the target frequency shown in Figure $7 \mathrm{~b}$, the LSCSA cannot extract the weak target signal, which proves the necessity of the SVD to further extract the target signal.

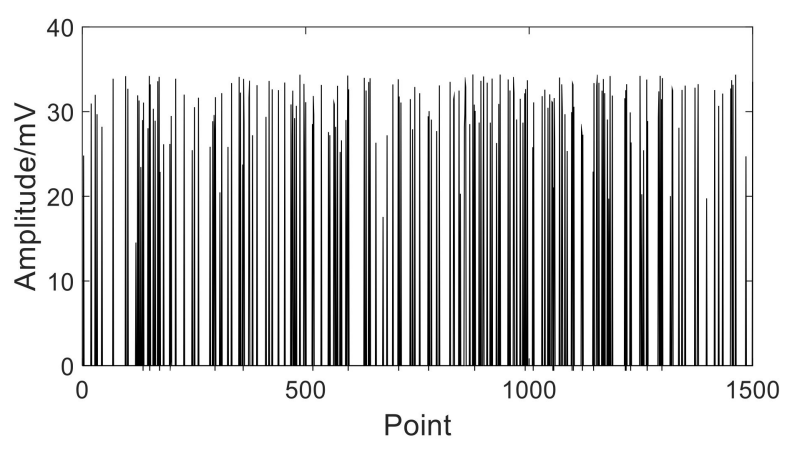

(a)

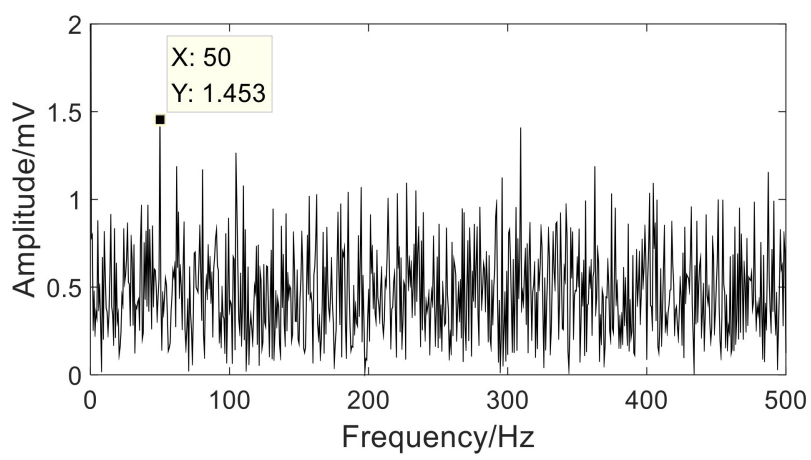

(b)

Figure 7. The results of LSCSA (a) Time domain (b) Frequency domain.

After using the LSCSA, the singular value vector of the simulation signal is shown in Figure 8. The three independent components of the signal are clearly shown in Figure 8. It proves the correctness of the theory in Section 2.3.

LSCSA-SVD is used to extract the target signal from the simulation signal. The time domain diagram, time domain local amplification diagram, and frequency domain diagram of the simulation results are shown in Figure 9.

In this paper, multiple autocorrelation, SVD, SCSA-SVD, and dual-tree complex wavelet transform (DTCWT), and singular value decomposition (SVD) are used as comparison methods. The time domain and frequency domain results of the comparison methods are shown in Figure 10. The SNR before and after using each method are shown in Table 1. 


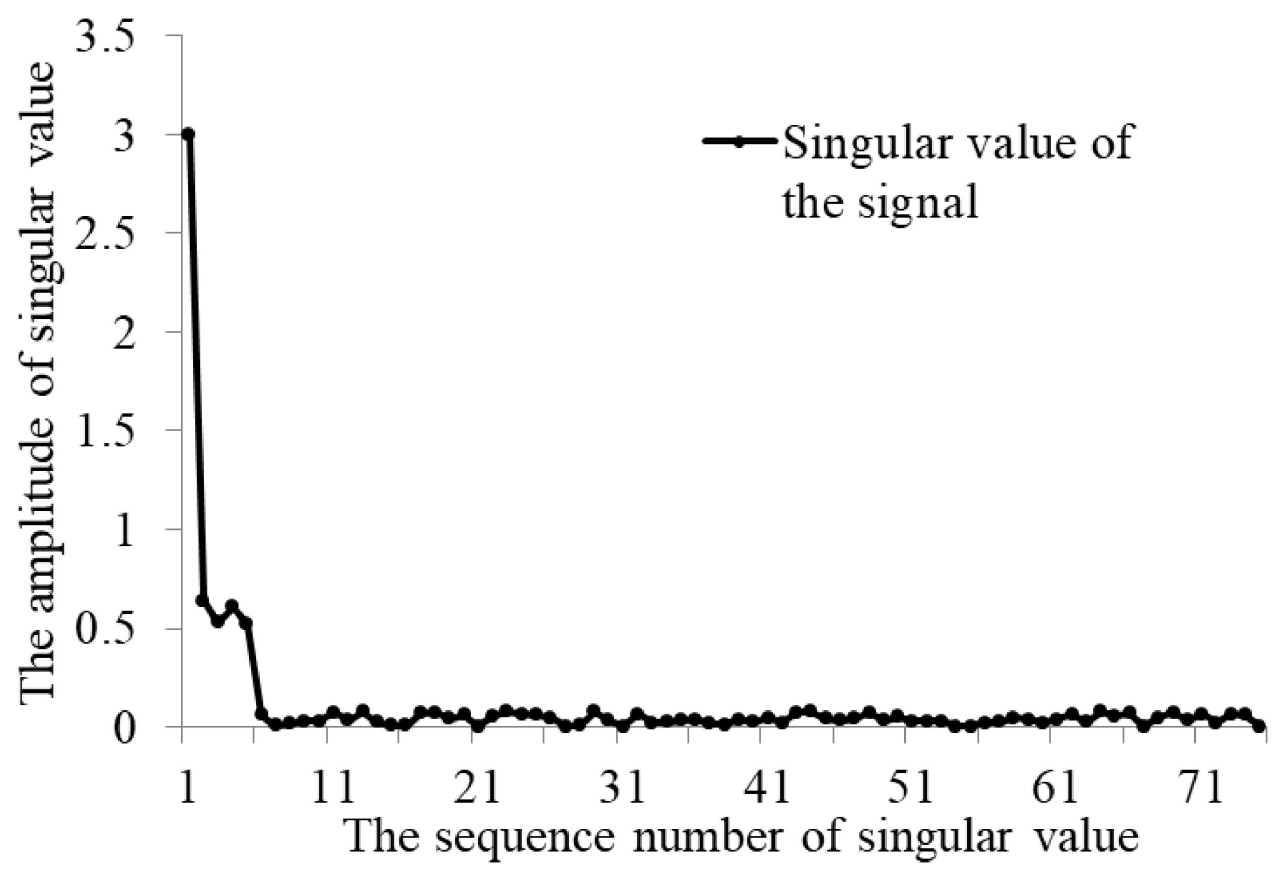

Figure 8. Singular value vector after using the LSCSA.

Multiple autocorrelation: Multiple autocorrelation is a traditional weak signal detection method. This example is used to test the performance of the LSCSA-SVD [18].

SVD: SVD is also a traditional weak signal detection algorithm [21]. This example not only verifies the performance of the LSCSA-SVD, but also proves the necessity of the LSCSA.

SCSA-SVD: This example is used to prove the necessity of the LSCSA.

DTCWT-SVD: DTCWT is used to carry out multilevel decomposition and purifying for an acceleration series. SVD is used to de-noise each layer of wavelet in the decomposition process. The decomposed signals are filtered and SVD denoised to detect weak signals [40].

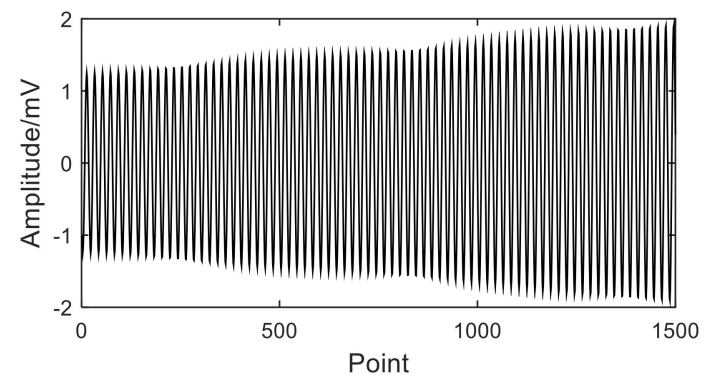

(a)

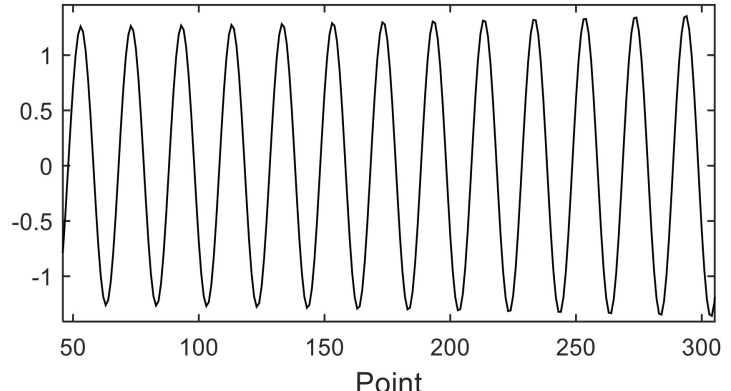

(b)

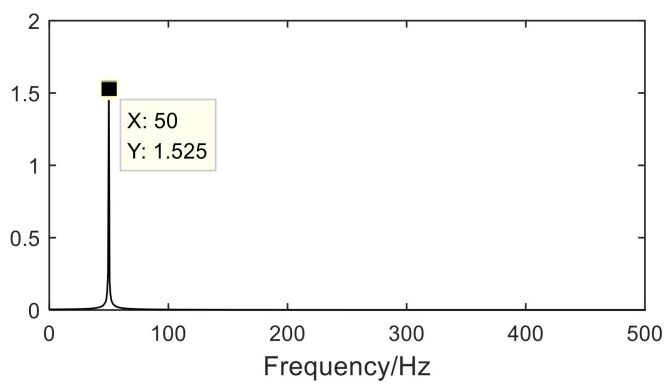

(c)

Figure 9. Simulation results of LSCSA-SVD (a) Time domain (b) Local amplification diagram of (a,c) Frequency domain. 
The spectrum diagram of the target signal in Figure 9c, with a peak frequency of $50 \mathrm{~Hz}$ and amplitude of $1.52 \mathrm{mV}$, is very close to the selected target signal.

In Figure 10a, although the target feature frequency is extracted, there are multiple prominent frequency peaks, which indicate that the detection accuracy of multiple autocorrelation is not high. Due to the limitation of autocorrelation theory, multiple autocorrelation cannot improve the weak signal.

By comparing Figures 9 and 10b, it is shown that the LSCSA can accurately locate the target signal. The LSCSA can effectively prevent target signals from being wrongly filtered and improve the detection success rate of weak signals. In Figure 10b,c, it is observed that the feature frequency is inconsistent with $50 \mathrm{~Hz}$, which indicates that SVD filters out the target signal. The simulation results are consistent with the theory in Section 2.3.
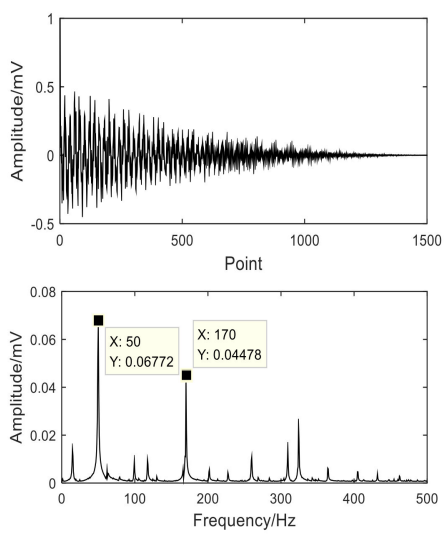

(a)
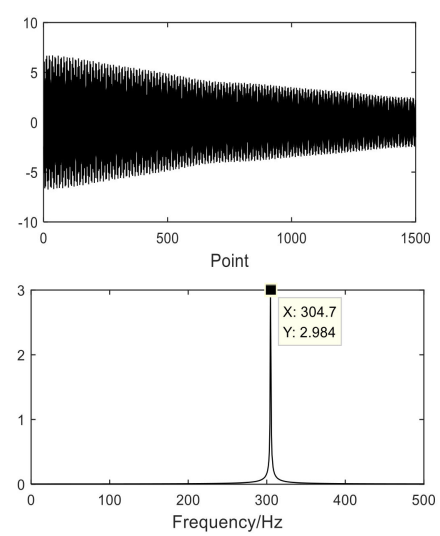

(b)
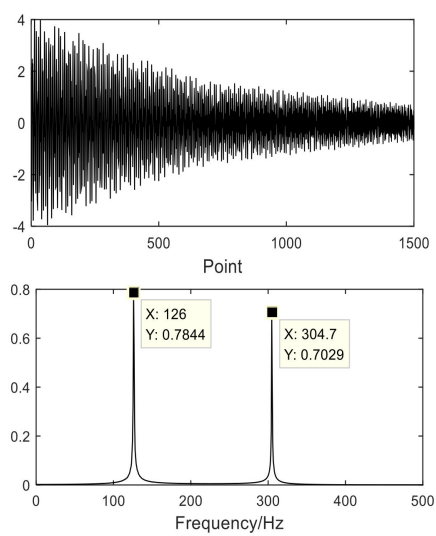

(c)
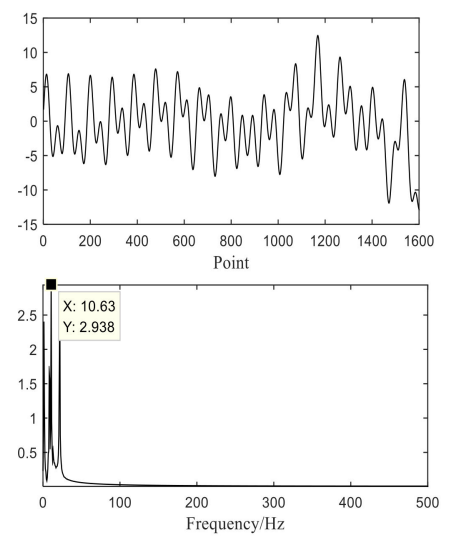

(d)

Figure 10. Simulation results of comparison methods. (Top: Time domain. Bottom: Frequency domain). (a) Multiple autocorrelation (b) SVD (c) SCSA-SVD (d) DTCWT-SVD.

Table 1. The SNR before and after using each method.

\begin{tabular}{cccccc}
\hline & LSCSA-SVD & $\begin{array}{c}\text { Multiple } \\
\text { Autocorrelation }\end{array}$ & SVD & SCSA-SVD & DTCWT-SVD \\
\hline Before & $-30 \mathrm{~dB}$ & $-30 \mathrm{~dB}$ & $-30 \mathrm{~dB}$ & $-30 \mathrm{~dB}$ & $-30 \mathrm{~dB}$ \\
After & $19.11 \mathrm{~dB}$ & $-0.06 \mathrm{~dB}$ & $-6.91 \mathrm{~dB}$ & $-5.46 \mathrm{~dB}$ & -7.34 \\
\hline
\end{tabular}

The results in Figure 10d show that when the noise intensity is very high, the target signal features cannot be enhanced even after DTCWT decomposition, which leads to the DTCWT-SVD not being able to extract the weak target signal. It shows the importance of enhancing the target signal features in weak signal detection and the effectiveness of the LSCSA in locating and enhancing the target signal.

Figure 10 indicates that the comparison methods cannot detect weak signals. In Table 1 , LSCSA-SVD has an obvious SNR improvement effect, while the comparison methods do not. To sum up, the LSCSA-SVD can significantly improve the SNR of weak signals and accurately extract weak target signals.

\section{Experimentation}

\subsection{The Weak Signal of Acceleration Sensor}

Rolling bearing failure is one of the main causes of failure in rotating machinery [41]. The rolling bearing fault signal is often submerged in the background of strong noise, which makes it difficult to extract its features. This case is mainly concerned with the algorithm's ability to extract fault features from strong noise, rather than early fault extraction.

The bearing condition monitoring system uses an acceleration sensor to monitor the bearing working condition in real time. The sensor is usually mounted on a bearing housing. As a result, the collected vibration signals are susceptible to pollution by various 
background noises. Especially when an early fault occurs, the weak fault feature signal is difficult to detect in time.

In order to verify the effect of LSCSA-SVD applied to sensor signals, the weak features of the rolling bearing fault signals collected in experiments were extracted. Experimental data from the rolling bearing database of Case Western Reserve University (CWRU) are used, and the deep groove ball bearing with 6205-2RS JEM SKF model is selected in this paper. The fault type is the outer ring bearing fault. The rolling bearing and experimental acquisition parameters are shown in Table 2.

Table 2. Rolling bearing and experimental acquisition parameters.

\begin{tabular}{ccccc}
\hline Inner Ring Diameter (D1) & Outer Ring Diameter (D2) & Thickness (h) & Ball Diameter (d) & Pitch Diameter (D) \\
\hline 0.9843 in & 2.0472 in & 0.5906 in & 0.3126 in & 1.537 in \\
\hline Ball number (n) & Contact angle $(\alpha)$ & $\begin{array}{c}\text { Sampling } \\
\text { Frequency (Fs) }\end{array}$ & Sampling Point & $\begin{array}{c}\text { Bearing Rotating } \\
\text { Speed (r) }\end{array}$ \\
\hline 9 & 0 & $12 \mathrm{kHz}$ & 20,000 & $1796 \mathrm{rpm}$ \\
\hline
\end{tabular}

The failure frequency of the rolling bearing outer ring can be calculated by Equation (13) [42]. The calculated fault frequency of the bearing outer ring is about $104.3 \mathrm{~Hz}$.

$$
f=\frac{r n}{120}\left(1-\frac{d}{D} \cos \alpha\right)
$$

In this paper, 1500 sampling points in the data are used for analysis. The time domain diagram and envelope spectrum diagram of the original data are shown in Figure 11.

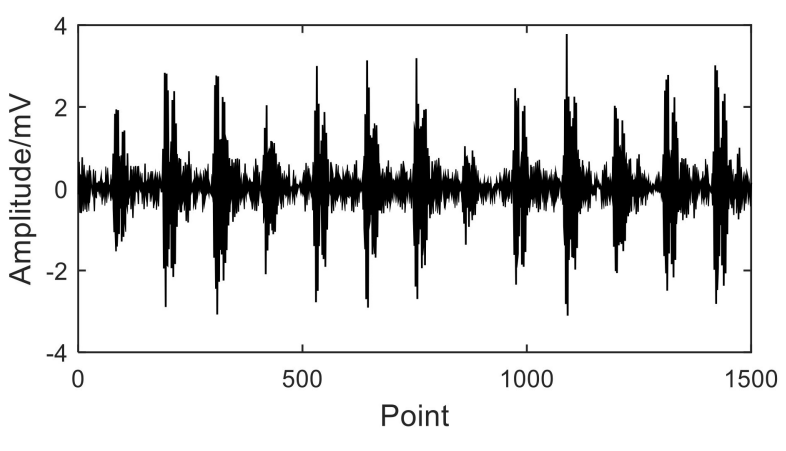

(a)

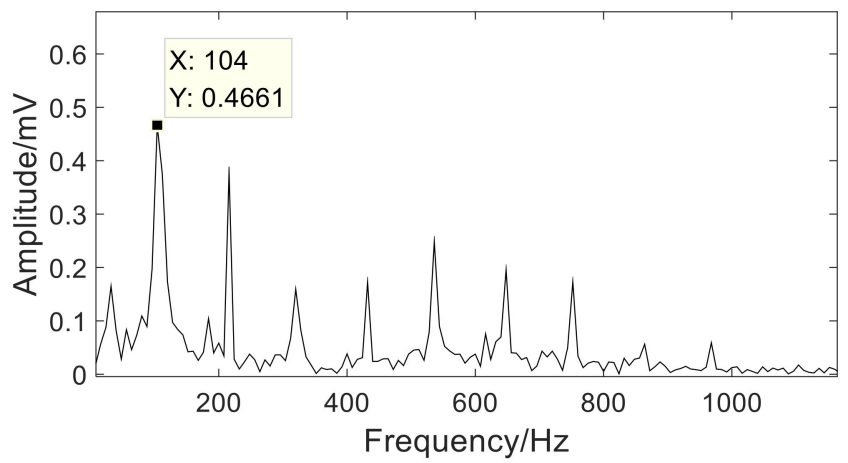

(b)

Figure 11. The original data (a) Time domain (b) Envelope spectrum.

The highest peak frequency, $108 \mathrm{~Hz}$, is the failure frequency of the bearing outer ring. The other peak frequencies are $208 \mathrm{~Hz}, 312 \mathrm{~Hz}, 416 \mathrm{~Hz}, 520 \mathrm{~Hz}, 624 \mathrm{~Hz}$, and $728 \mathrm{~Hz}$, which are the two to seven times failure frequency of the bearing outer ring. Since the noise of the CWRU experimental data is relatively weak, Gaussian white noise (SNR $=-30 \mathrm{~dB}$ ) is added to the experimental data in this paper. The time domain diagram and envelope spectrum of experimental signals with noise are shown in Figure 12.

The highest peak in Figure 12b appears at $264 \mathrm{~Hz}$, which is not the bearing outer ring failure frequency. It indicates that the bearing outer ring failure frequency has been completely annihilated in the strong noise, which is a weak target signal to be extracted in the experiment.

LSCSA-SVD is used to extract the experimental signals with noise. Select parameter $h=119.3$ and parameter $k=1253$. The envelope spectrum of the weak target signal extracted is shown in Figure 13. 


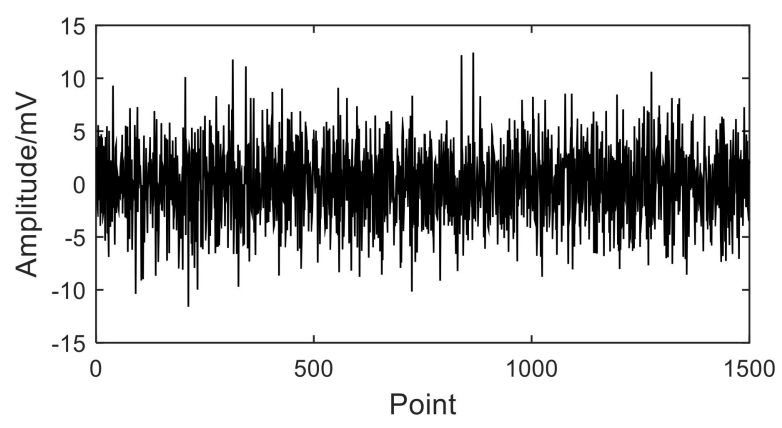

(a)

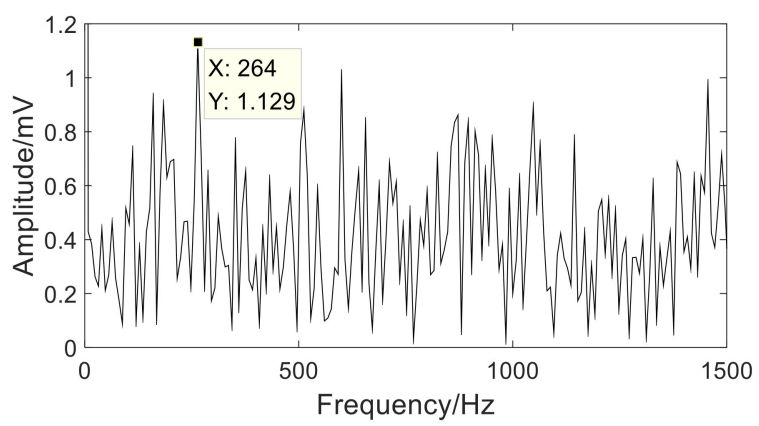

(b)

Figure 12. Experimental signals with noise (a) Time domain (b) Envelope spectrum.

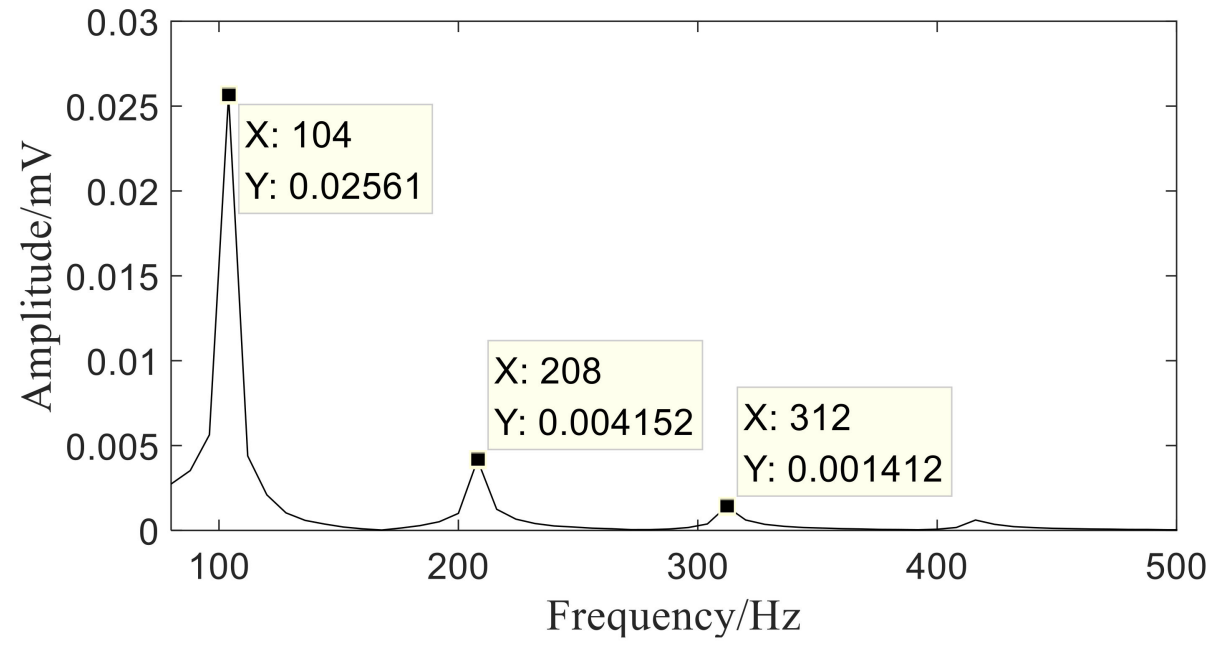

Figure 13. Envelope spectrum of LSCSA-SVD.

According to Figure 13, the peak frequency is $104 \mathrm{~Hz}$, which is equal to the bearing outer ring failure frequency. This indicates that this signal is the weak target signal required by the experiment, and the strong noise has been eliminated. There are also double and triple frequencies in Figure 13, which means that LSCSA not only eliminates noise, but also retains the bearing fault features completely. This shows that LSCSA-SVD can be applied to pulse signals. The above are proof that the LSCSA-SVD can suppress strong noise effectively and can be used to extract weak bearing signals.

In the experiment, DTCWT-SVD and SCSA-SVD are used as comparison weak signal detection methods. The comparison methods are used to extract the experimental signals with noise, and the envelope spectrum of the extracted results is shown in Figure 14.

According to Figure 14, under the interference of strong noise, neither of the two comparison methods extracted the target signal. This indicates that the proposed method is superior to the traditional weak signal detection method.

To sum up, the LSCSA-SVD algorithm can accurately extract weak target signals in sensor signals. The method proposed in this paper is a very effective method for weak signal detection. 


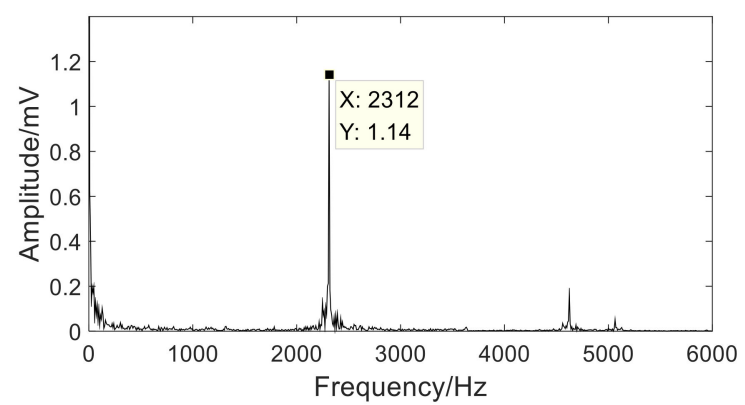

(a)

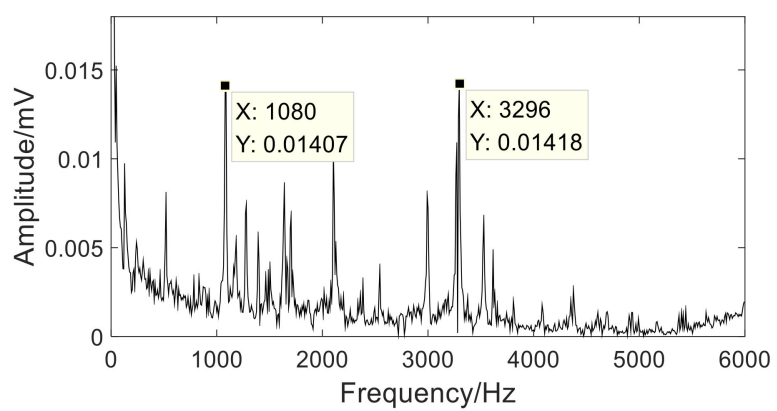

(b)

Figure 14. Envelope spectrum of the comparison methods (a) DTCWT-SVD (b) SCSA-SVD.

\subsection{The Weak Signal of Test Circuit}

This experiment aims to test the application effect of the LSCSA in the field of instruments. The equipment of the test system includes a signal generator, a weak signal analog amplifier circuit board, a signal acquisition instrument, a shielding box, and a computer. In signal generation, a target signal with a frequency of $179.2 \mathrm{~Hz}$ and a peak-to-peak value of $20 \mathrm{mVpp}$ is generated. At the same time, a clock signal with a frequency of $30 \mathrm{kHz}$ and a duty cycle of $10 \%$ is generated. The target signal is attenuated by a large capacitor, and the amplification results are output by a weak signal analog amplifier circuit board. A signal acquisition instrument is used to collect the output signal of the amplifying chip. A shielding box is used to shield outside interference. The test system and analog amplifier circuit board are shown in Figure 15. The time domain and frequency domain diagrams of the signal collected by the test system are shown in Figure 16.

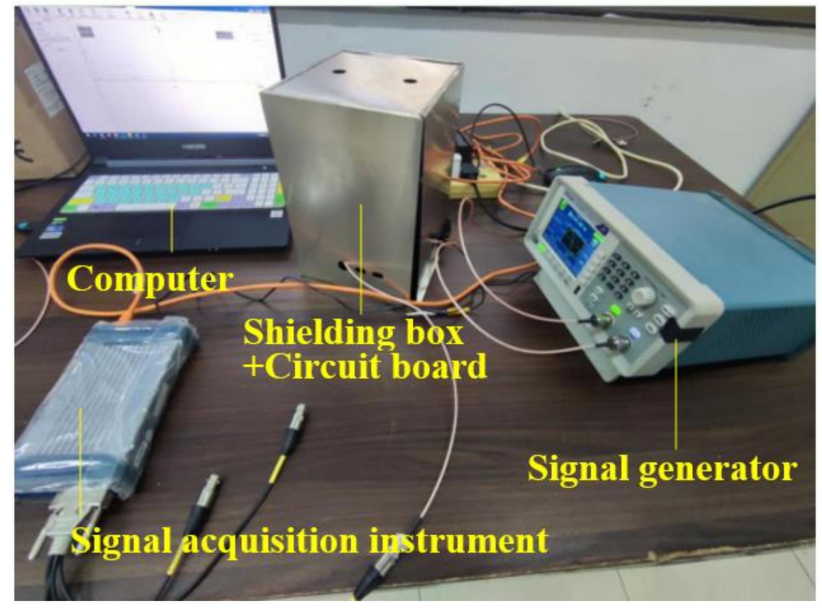

(a)

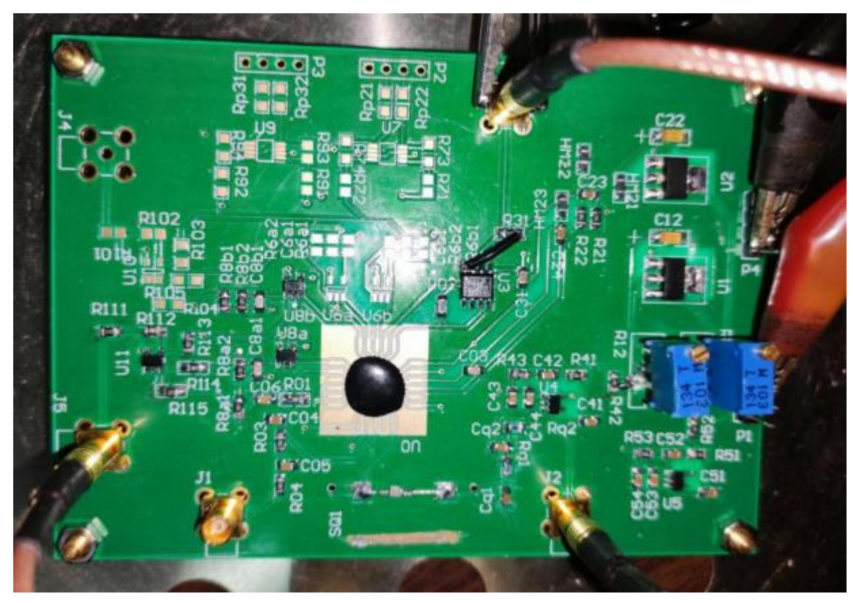

(b)

Figure 15. The test system and analog amplifier circuit board (a) The test system (b) The analog amplifier circuit board.

Figure 16b shows the target frequency of $179.2 \mathrm{~Hz}$, the high frequency of $7501 \mathrm{~Hz}$, and its multiplier. The target signal is very weak. The LSCSA-SVD is used to extract weak target signals. The time domain and frequency domain diagrams of the extraction results are shown in Figure 17. 


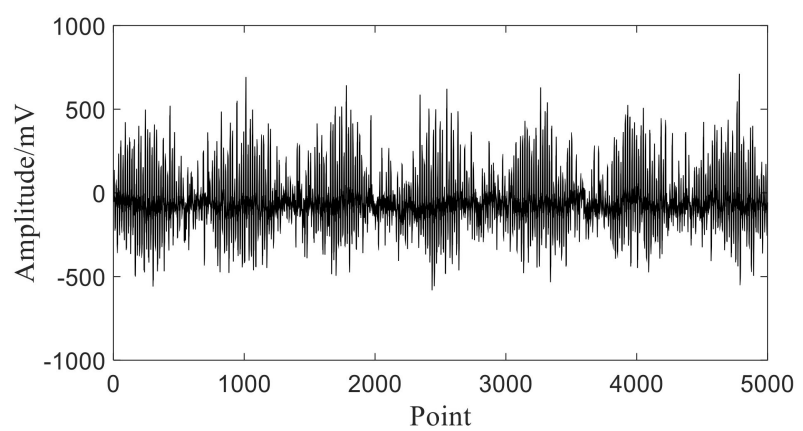

(a)

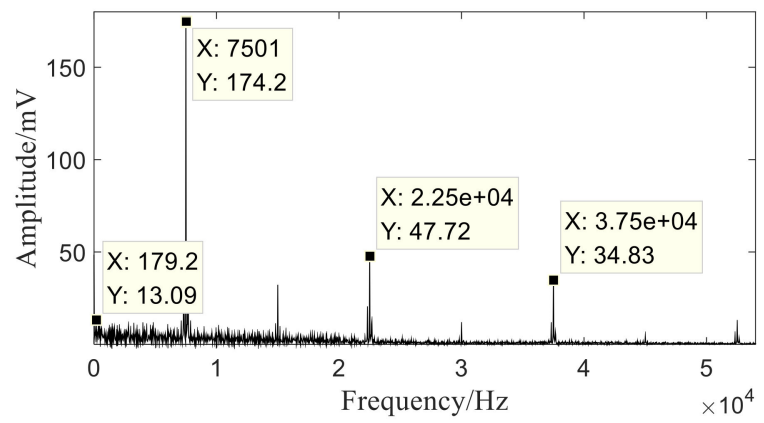

(b)

Figure 16. Data collected by the test system (a) Time domain (b) Frequency domain.

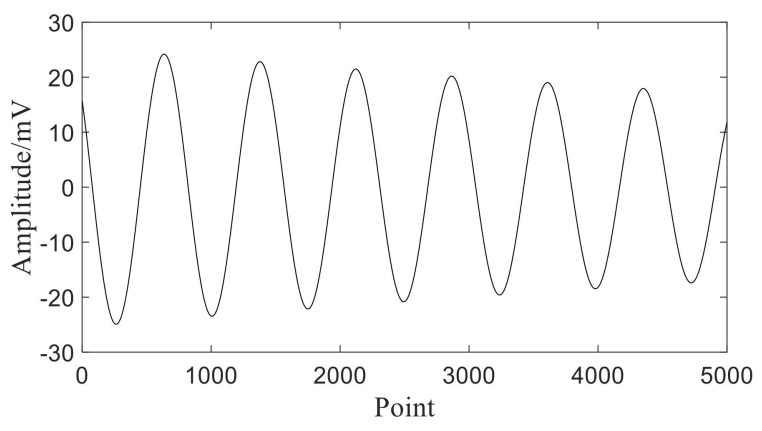

(a)

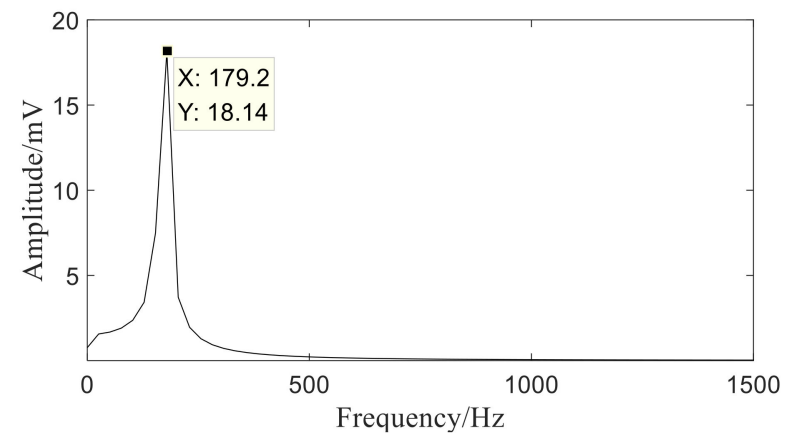

(b)

Figure 17. The extraction results of LSCSA-SVD (a) Time domain (b) Frequency domain.

In Figure 17, the target signal has been successfully extracted, and the amplitude of the extracted signal is very close to the amplitude set by the signal generator, which indicates that the LSCSA has a very good application effect on the signal collected by the test system and can achieve strong noise suppression. The LSCSA proposed in this paper can be applied to the field of instruments.

\section{Conclusions}

A quantum weak signal detection method, Local Semi-Classical Signal AnalysisSingular Value Decomposition (LSCSA-SVD), is proposed. Firstly, the LSCSA is used to quantize the time domain weak signal. In the quantum domain, the weak target signal mainly exists in the later portion of the eigenvalue sequence (detail region). The weak target signal can be located in strong noise only by using the detailed region to reconstruct the signal. Then, the reconstruction signal is decomposed by using SVD. In the singular value vector, the singular values representing the weak target signal and the strong noise signal components can be separated as separate coordinates. By this characteristic, noise and target signals can be accurately separated.

The results in Section 4 show that the weak signal features are protected and strengthened by LSCSA, which solves the problem that SVD cannot detect weak signals when the signal features are not obvious. SVD is used to further extract the signal features of the LSCSA reconstruction signal, which solves the problem that LSCSA cannot extract signal features directly. The results in Sections 4 and 5 show that LSCSA-SVD can detect weak signals with low SNR and improve the SNR, and that it cannot be achieved by using LSCSA and SVD alone. The LSCSA-SVD is suitable for the field of mechanics and the field of instruments.

In future work, we will further study how to improve the algorithm speed and automatic adjustment of algorithm parameters. 
Author Contributions: Conceptualization, T.Y.; methodology, T.Y. and S.G.; software, T.Y.; validation, T.Y., S.L. and J.L.; formal analysis, T.Y.; investigation, T.Y.; resources, T.Y., S.L. and J.G.; data curation, T.Y.; writing—original draft preparation, T.Y.; writing—review and editing, T.Y.; visualization, T.Y., J.L. and Y.C.; supervision, S.L., J.L., J.G. and Y.C.; project administration, S.L.; funding acquisition, S.L. and J.G. All authors have read and agreed to the published version of the manuscript.

Funding: This research was funded by the National Key Research and Development Program of China (2018YFB2003300), and the National Natural Science Foundation of China (51975276).

Institutional Review Board Statement: Not applicable.

Informed Consent Statement: Not applicable.

Data Availability Statement: Publicly available datasets were analyzed in this study. This data can be found here: [https:/ / engineering.case.edu/bearingdatacenter] (accessed on 19 December 2021).

Conflicts of Interest: The authors declare no conflict of interest.

\section{References}

1. Gao, J. Weak Signal Detection; Tsinghua University Press: Beijing, China, 2004.

2. Kikuchi, S.; Takeuchi, H.; Mori, O.; Kato, H.; Ogawa, N.; Mimasu, Y.; Tomiki, A.; Taniguchi, S. Off-Line Signal Processing for Weak-Signal Detection and Orbit and Attitude Determination. J. Spacecr. Rocket. 2019, 56, 117-128. [CrossRef]

3. Wang, X.; Liu, G.; Xu, M.; Ren, B.; Tian, Z. Development of Weak Signal Recognition and Extraction Algorithm for Fast Raman Imaging. Anal. Chem. 2019, 91, 12909-12916. [CrossRef] [PubMed]

4. Tandra, R.; Sahai, A. SNR Walls for Signal Detection. IEEE J. Sel. Top. Signal Process. 2008, 2, 4-17. [CrossRef]

5. Li, Z.; Zhang, P.; Mu, X.; Jia, P.; Hu, Y.; Chen, Z.; Xu, J. Guiding and routing of a weak signal via a reconfigurable gravity-like potential. Photonics Res. 2019, 7, 1087-1093. [CrossRef]

6. Chowdhury, A.; Clerc, M.G.; Barbay, S.; Robert-Philip, I.; Braive, R. Weak signal enhancement by nonlinear resonance control in a forced nano-electromechanical resonator. Nat. Commun. 2020, 11, 1-9. [CrossRef] [PubMed]

7. Kerstens, R.; Schouten, G.; Jansen, W.; Laurijssen, D.; Steckel, J. Live Demonstration of eRTIS, an Embedded Real-Time Imaging Sonar Sensor. In Proceedings of the 2019 IEEE Sensors, Montreal, QC, Canada, 27-30 October 2019. [CrossRef]

8. Chen, W.; Gu, K.; Lin, W.; Xia, Z.; Le Callet, P.; Cheng, E. Reference-Free Quality Assessment of Sonar Images via Contour Degradation Measurement. IEEE Trans. Image Process. 2019, 28, 5336-5351. [CrossRef] [PubMed]

9. Li, J.; Zhang, J.; Li, M.; Zhang, Y. A novel adaptive stochastic resonance method based on coupled bistable systems and its application in rolling bearing fault diagnosis. Mech. Syst. Signal Process. 2019, 114, 128-145. [CrossRef]

10. Shijie, S.; Kai, W.; Xuliang, Q.; Dan, Z.; Xueqing, D.; Jiale, S. Investigation on Bearing Weak Fault Diagnosis under Colored Noise. In Proceedings of the 2020 Chinese Control and Decision Conference (CCDC), Hefei, China, 22-24 August 2020.

11. Kokil, P.; Pratap, T. Additive White Gaussian Noise Level Estimation for Natural Images Using Linear Scale-Space Features. Circuits Syst. Signal Process. 2020, 40, 353-374. [CrossRef]

12. Fatnan, M.H.; Hussain, Z.; Mohammed, H.R. Blind Source Separation under Semi-White Gaussian Noise and Uniform Noise: Performance Analysis of ICA, Sobi and JadeR. J. Comput. Sci. 2019, 15, 27-44. [CrossRef]

13. Wang, Z.; Shi, X. Electric activities of time-delay memristive neuron disturbed by Gaussian white noise. Cogn. Neurodyn. 2019, 14, 115-124. [CrossRef]

14. Guo, L.; Kan, E.; Wu, Y.; Lv, H.; Xu, G. Noise suppression ability and its mechanism analysis of scale-free spiking neural network under white Gaussian noise. PLoS ONE 2020, 15, e0244683. [CrossRef]

15. Aquino, G.; Rocco, A. Bimodality in gene expression without feedback: From Gaussian white noise to log-normal coloured noise. Math. Biosci. Eng. 2020, 17, 6993-7071. [CrossRef]

16. Zabala-Blanco, D.; Azurdia-Meza, C.A.; Montejo-Sánchez, S.; Firoozabadi, A.D. OFDM tolerance to additive white Gaussian and laser phase noises in optical heterodyning systems accompanied by the feasible pilot-assisted equalization. Opt. Rev. 2020, 27, 156-169. [CrossRef]

17. Al-Azzawi, A.K. High Empirical Study of Edge Detection-Based Image Denoising Corrupted by the Additive White Gaussian Noise (WGN). IOP Conf. Ser. Mater. Sci. Eng. 2020, 881, 012106. [CrossRef]

18. Elasmi-Ksibi, R.; Besbes, H.; López-Valcarce, R.; Cherif, S. Frequency estimation of real-valued single-tone in colored noise using multiple autocorrelation lags. Signal Process. 2010, 90, 2303-2307. [CrossRef]

19. He, X.; Liu, Q.; Yu, W.; Mechefske, C.K.; Zhou, X. A new autocorrelation-based strategy for multiple fault feature extraction from gearbox vibration signals. Measurement 2020, 171, 108738. [CrossRef]

20. Zhao, X.; Ye, B. Singular value decomposition packet and its application to extraction of weak fault feature. Mech. Syst. Signal Process. 2016, 70, 73-86. [CrossRef]

21. Hou, F.; Chen, J.; Dong, G. Weak fault feature extraction of rolling bearings based on globally optimized sparse coding and approximate SVD. Mech. Syst. Signal Process. 2018, 111, 234-250. [CrossRef] 
22. Lei, Y.; Yang, B.; Jiang, X.; Jia, F.; Li, N.; Nandi, A.K. Applications of machine learning to machine fault diagnosis: A review and roadmap. Mech. Syst. Signal Process. 2020, 138, 106587. [CrossRef]

23. Sun, J.; Yan, C.; Wen, J. Intelligent Bearing Fault Diagnosis Method Combining Compressed Data Acquisition and Deep Learning IEEE Trans. Instrum. Meas. 2017, 67, 185-195. [CrossRef]

24. Yildirim, A.; Kiranyaz, S. 1D Convolutional Neural Networks Versus Automatic Classifiers for Known LPI Radar Signals Under White Gaussian Noise. IEEE Access 2020, 8, 180534. [CrossRef]

25. Eldar, Y.C.; Oppenheim, A.V. Quantum signal processing. IEEE Signal Process. Mag. 2001, 19, 12-32. [CrossRef]

26. Chapeau-Blondeau, F.; Belin, E. Fourier-transform quantum phase estimation with quantum phase noise. Signal Process. 2019, 170, 107441. [CrossRef]

27. Le, Y. Vibration Signal Processing of Transmission System Based on Quantum Inspired Mathematical Morphology. Mech. Transm. 2017, 41, 189-193.

28. Pang, C.Y.; Hu, B.Q. Quantum Discrete Fourier Transform with Classical Output for Signal Processing. Physics 2007, 101, 309-332.

29. Yanlong, C.; Peilin, Z.; Huaiguang, W. Mechanical Vibration Signal Denoising Method Based on Quantum Superposition State Parameter Estimation. Shock. Vib. 2014, 33, 143-147.

30. Laleg-Kirati, T.M.; Crépeau, E.; Sorine, M. Semi-classical signal analysis. Math. Control. Signals Syst. 2013, 25, 37-61. [CrossRef]

31. Smith, R.; Basarab, A.; Georgeot, B.; Kouame, D. Adaptive transform via quantum signal processing: Application to signal and image denoising. In Proceedings of the 2018 25th IEEE International Conference on Image Processing (ICIP), Athens, Greece, 7-10 October 2018; pp. 1523-1527. [CrossRef]

32. Low, G.H.; Chuang, I.L. Optimal Hamiltonian Simulation by Quantum Signal Processing. Phys. Rev. Lett. 2016, 118, 010501. [CrossRef]

33. Nieto-Chaupis, H. Quantum Effects without Quantum Fields: Feynman's Amplitudes in Classical Electrodynamics. In Proceedings of the 2019 IEEE 2nd British and Irish Conference on Optics and Photonics (BICOP), London, UK, 11-13 December 2019; pp. 1-4. [CrossRef]

34. Wen, T.; Yan, J.; Huang, D.; Lu, K.; Deng, C.; Zeng, T.; Yu, S.; He, Z. Feature Extraction of Electronic Nose Signals Using QPSO-Based Multiple KFDA Signal Processing. Sensors 2018, 18, 388. [CrossRef]

35. Bhaduri, S.; Chahid, A.; Achten, E.; Laleg-Kirati, T.M.; Serrai, H. SCSA based MATLAB pre-processing toolbox for 1H MR spectroscopic water sup-pression and denoising. Inform. Med. Unlocked 2020, 18, 100294. [CrossRef]

36. Laleg-Kirati, T.M.; Zhang, J.; Achten, E.; Serrai, H. Spectral data de-noising using semi-classical signal analysis: Application to lo-calized MRS. NMR Biomed. 2016, 29, 1477-1485. [CrossRef]

37. Chahid, A.; Serrai, H.; Achten, E.; Laleg-Kirati, T.M. A New ROI-Based performance evaluation method for image denoising using the Squared Eigenfunctions of the Schrödinger Operator. In Proceedings of the 2018 40th Annual International Conference of the IEEE Engineering in Medicine and Biology Society (EMBC), Honolulu, HI, USA, 18-21 July 2018; Volume 2018, pp. 5579-5582. [CrossRef]

38. Zhang, X. Efficient Solution of Matlab Differential Equation: Principle and Implementation of Spectral Method; Mechanical Industry Press: Beijing, China, 2016.

39. Zhao, X.; Ye, B.; Chen, J. Weak fault Feature Extraction Method based on Wavelet singular value Decomposition Differential spectrum. J. Mech. Eng. 2012, 7, 37-48. [CrossRef]

40. Wang, N.F.; Jiang, D.X.; Yang, W.G. Dual-Tree Complex Wavelet Transform and SVD-Based Acceleration Signals Denoising and its Application in Fault Features Enhancement for Wind Turbine. J. Vib. Eng. Technol. 2019, 7, 311-320. [CrossRef]

41. Wang, H.; Chen, J.; Dong, G. Feature extraction of rolling bearing's early weak fault based on EEMD and tunable Q-factor wavelet transform. Mech. Syst. Signal Process. 2014, 48, 103-119. [CrossRef]

42. Zhao, X.; Ye, B. Similarity of signal processing effect between Hankel matrix-based SVD and wavelet transform and its mechanism analysis. Mech. Syst. Signal Process. 2009, 23, 1062-1075. [CrossRef] 